\title{
The Cosmological Constant from the Extended Theory of Gravitation in Clifford Spaces
}

\author{
Carlos Castro
}

October, 2014

Center for Theoretical Studies of Physical Systems, Clark Atlanta University, Atlanta; perelmanc@hotmail.com

\begin{abstract}
The exploration of the novel physical consequences of the Extended Theory of Gravity in $C$-spaces (Clifford spaces) is continued. One of the most salient physical feature of the extended gravitational theory in $C$-spaces is that one can generate an effective stress energy tensor mimicking the effects of "dark" matter/energy. In particular, it is found that the presence of the cosmological constant, along with a plausible mechanism to explain its extremely small value and/or its cancellation, can be understood entirely from a purely Clifford algebraic and geometric perspective. For this reason we believe that this theory may have important consequences in Cosmology and further research in Gravitation and Particle Physics.
\end{abstract}

Keywords : Clifford algebras; Extended Relativity in Clifford Spaces; Cosmological Constant, Cosmology.

\section{Introduction : C-space Relativity}

In the past years, the Extended Relativity Theory in $C$-spaces (Clifford spaces) and Clifford-Phase spaces were developed [1], [2]. The Extended Relativity theory in Cliffordspaces (C-spaces) is a natural extension of the ordinary Relativity theory whose generalized coordinates are Clifford polyvector-valued quantities which incorporate the lines, areas, volumes, and hyper-volumes degrees of freedom associated with the collective dynamics of particles, strings, membranes, p-branes (closed p-branes) moving in a D-dimensional target spacetime background. C-space Relativity permits to study the dynamics of all (closed) p-branes, for different values of $\mathrm{p}$, on a unified footing. 
Our theory has 2 fundamental parameters : the speed of a light $c$ and a length scale which can be set equal to the Planck length. The role of "photons" in $C$-space is played by tensionless branes. An extensive review of the Extended Relativity Theory in Clifford spaces can be found in [1]. The polyvector valued coordinates $x^{\mu}, x^{\mu_{1} \mu_{2}}, x^{\mu_{1} \mu_{2} \mu_{3}}, \ldots$ are now linked to the basis vectors generators $\gamma^{\mu}$, bi-vectors generators $\gamma_{\mu} \wedge \gamma_{\nu}$, tri-vectors generators $\gamma_{\mu_{1}} \wedge \gamma_{\mu_{2}} \wedge \gamma_{\mu_{3}}$, ... of the Clifford algebra, including the Clifford algebra unit element (associated to a scalar coordinate).

These polyvector valued coordinates can be interpreted as the quenched-degrees of freedom of an ensemble of $p$-loops associated with the dynamics of closed $p$-branes, for $p=0,1,2, \ldots, D-1$, embedded in a target $D$-dimensional spacetime background. $C$-space is parametrized not only by 1 -vector coordinates $x^{\mu}$ but also by the 2 -vector coordinates $x^{\mu \nu}, 3$-vector coordinates $x^{\mu \nu \alpha}, \ldots$, called also holographic coordinates, since they describe the holographic projections of 1-loops, 2-loops, 3-loops,..., onto the coordinate planes . By $p$-loop we mean a closed $p$-brane; in particular, a 1-loop is closed string. When $\mathbf{X}$ is the Clifford-valued coordinate corresponding to the $C l(1,3)$ algebra in four-dimensions it can be decomposed as

$$
\mathbf{X}=s \mathbf{1}+x^{\mu} \gamma_{\mu}+x^{\mu \nu} \gamma_{\mu} \wedge \gamma_{\nu}+x^{\mu \nu \rho} \gamma_{\mu} \wedge \gamma_{\nu} \wedge \gamma_{\rho}+x^{\mu \nu \rho \tau} \gamma_{\mu} \wedge \gamma_{\nu} \wedge \gamma_{\rho} \wedge \gamma_{\tau}
$$

where we have omitted combinatorial numerical factors for convenience in the expansion of eq-(1.1). To avoid introducing powers of a length parameter $L$ (like the Planck scale $\left.L_{p}\right)$, in order to match physical units in the expansion of the polyvector $\mathbf{X}$ in eq-(1.1), we can set it to unity to simplify matters.

The component $s$ is the Clifford scalar component of the polyvector-valued coordinate and $d \Sigma$ is the infinitesimal $C$-space proper "time" interval

$$
(d \Sigma)^{2}=(d s)^{2}+d x_{\mu} d x^{\mu}+d x_{\mu \nu} d x^{\mu \nu}+\ldots
$$

that is invariant under $C l(1,3)$ transformations and which are the Clifford-algebraic extensions of the $S O(1,3)$ Lorentz transformations [1]. One should emphasize that $d \Sigma$ is not equal to the proper time Lorentz-invariant interval $d \tau$ in ordinary spacetime $(d \tau)^{2}=$ $g_{\mu \nu} d x^{\mu} d x^{\nu}=d x_{\mu} d x^{\mu}$. Generalized Lorentz transformations (poly-rotations) in flat $C$ spaces were discussed in [1]. A recent extensive analysis of the $C$-space generalized Lorentz transformations and their physical implications can be found in [9].

Given $\mathbf{X}=X^{A} \gamma_{A}$, where $A$ is a polyvector-valued index and $\gamma_{A}$ span over all the generators of the Clifford algebra, the quadratic form is defined as

$$
<\mathbf{X}^{\dagger} \mathbf{X}>=X_{A} X^{A}=s^{2}+X_{\mu} X^{\mu}+X_{\mu_{1} \mu_{2}} X^{\mu_{1} \mu_{2}}+\ldots \ldots X_{\mu_{1} \mu_{2} \ldots . \mu_{D}} X^{\mu_{1} \mu_{2} \ldots \mu_{D}}
$$

where $\mathbf{X}^{\dagger}$ denotes the reversal operation obtained by reversing the order of the gamma generators in the wedge products. The symbol $<\gamma_{A} \gamma_{B}>$ denotes taking the scalar part in the Clifford geometric product of $\gamma_{A} \gamma_{B}$. It is the analog of taking the trace of a product of matrices. Such scalar part can be obtained from the (anti) commutator relations of the Clifford algebra generators as displayed in the Appendix A. For example 


$$
\begin{gathered}
<\gamma_{\mu} \gamma^{\nu}>=\delta_{\mu}^{\nu}, \quad<\gamma_{\mu_{1} \mu_{2}} \gamma^{\nu_{1} \nu_{2}}>=-\delta_{\mu_{1} \mu_{2}}^{\nu_{1} \nu_{2}} \\
\left.<\gamma_{\mu_{1} \mu_{2} \mu_{3}} \gamma^{\nu_{1} \nu_{2} \nu_{3}}>=-\delta_{\mu_{1} \mu_{2} \mu_{3} \nu_{2} \nu_{3}}\right\rangle=\gamma_{\mu_{1} \mu_{2} \mu_{3} \mu_{4}} \gamma^{\nu_{1} \nu_{2} \nu_{3} \nu_{4}}>=\delta_{\mu_{1} \mu_{2} \mu_{3} \mu_{4}}^{\nu_{1} \nu_{2} \nu_{4}},
\end{gathered}
$$

One should note the presence of \pm signs in the right hand side of eqs-(1.4). They are connected to the even/odd behavior of the reversal operation $\left(\gamma_{C}\right)^{\dagger}= \pm \gamma_{C}$.

The quadratic form is invariant under the isometry transformations

$$
\mathbf{X}^{\prime}=\mathbf{R} \mathbf{X ~ L}^{\dagger}, \quad \mathbf{R}^{\dagger} \mathbf{R}=1, \quad \mathbf{L}^{\dagger} \mathbf{L}=1 \Rightarrow<\mathbf{X}^{\prime \dagger} \mathbf{X}^{\prime}>=<\mathbf{X}^{\dagger} \mathbf{X}>
$$

due to the cyclic property of the scalar part projection

$$
\begin{aligned}
<\mathbf{X}^{\prime \dagger} \mathbf{X}^{\prime}>= & <\mathbf{L} \mathbf{X}^{\dagger} \mathbf{R}^{\dagger} \mathbf{R} \mathbf{X} \mathbf{L}^{\dagger},>=<\mathbf{L} \mathbf{X}^{\dagger} \mathbf{X} \mathbf{L}^{\dagger}>= \\
& <\mathbf{L}^{\dagger} \mathbf{L} \mathbf{X}^{\dagger} \mathbf{X}==<\mathbf{X}^{\dagger} \mathbf{X}>
\end{aligned}
$$

where $\mathbf{R}, \mathbf{L}$ are Clifford-valued rotors acting on the right and left respectively. Let us write the rotor associated with a "rotation" along the $X^{A}-X^{B}$ directions in $C$-space with parameter $\alpha^{A B}$, after writing the commutation relations $\left[\Gamma_{A}, \Gamma_{B}\right]=f_{A B}{ }_{C} \Gamma_{C}$, as follows

$$
\mathbf{R}=e^{\alpha^{A B}\left[\Gamma_{A}, \Gamma_{B}\right]}=e^{\alpha^{A B}} f_{A B}^{C} \Gamma_{C}=e^{\beta^{C} \Gamma_{C}}, \beta^{C}=\alpha^{A B} f_{A B}^{C}
$$

where $f_{A B} C$ are the structure constants of the algebra. There is a summation over the $C$ indices (but not over the $A, B$ indices) in eq-(1.7) and the reversal condition reads

$$
\left[\Gamma_{A}, \Gamma_{B}\right]^{\dagger}=-\left[\Gamma_{A}, \Gamma_{B}\right] \Rightarrow \mathbf{R} \mathbf{R}^{\dagger}=1
$$

and which is satisfied in particular when $\Gamma_{A}^{\dagger}=-\Gamma_{A} ; \Gamma_{B}^{\dagger}=-\Gamma_{B}$ giving $\Gamma_{C}^{\dagger}=-\Gamma_{C}$. This is a result of the relations $\left(\Gamma_{A} \Gamma_{B}\right)^{\dagger}=\left(\Gamma_{B}\right)^{\dagger}\left(\Gamma_{A}\right)^{\dagger}=\Gamma_{B} \Gamma_{A}$. In the most general case, for arbitrary dimensions, due to the summation over the $C$ polyvector indices in eq-(1.7), the rotor $\mathbf{R}$ does not factorize into separate products. For instance, after performing a Taylor series expansion of the exponentials, one has that

$$
e^{\beta^{01} \gamma_{01}+\beta^{023} \gamma_{023}} \neq\left(\cosh \left(\beta^{01}\right)+\gamma_{01} \sinh \left(\beta^{01}\right)\right)\left(\cosh \left(\beta^{023}\right)+\gamma_{023} \sinh \left(\beta^{023}\right)\right)
$$

as a result of the Baker-Campbell-Hausdorf formula. Because $\left[\gamma_{01}, \gamma_{023}\right] \neq 0$ the left hand side of eq-(1.9) does not factorize. A detailed study (with several examples) of the generalized Lorentz transformations in $C$-space can be found in [9]. In particular when the polyvector coordinate components of different grades become entangled under the $C$-space generalized Lorentz transformations. Having presented this short but concise introduction of the notion of $C$-space we proceed with the crux of this work. For references on Clifford algebras and the Clifford approach to geometry see [3], [4], [7] [5], [6] and references therein. 


\section{The Differential Geometry of Curved $C$-spaces}

In curved $C$-space [1], [7] one introduces the $\mathbf{X}$-dependent basis generators $\gamma_{M}, \gamma^{M}$ defined in terms of the beins $E_{M}^{A}$, inverse beins $E_{A}^{M}$ and the flat tangent space generators $\gamma_{A}, \gamma^{A}$ as follows $\gamma_{M}=E_{M}^{A}(\mathbf{X}) \gamma_{A}, \gamma^{M}=E_{A}^{M}(\mathbf{X}) \gamma^{A}$. The curved $C$ - space metric expression $g_{M N}=E_{M}^{A} E_{N}^{B} \eta_{A B}$ also agrees with taking the scalar part of the Clifford geometric product $<\gamma_{M} \gamma_{N}>=g_{M N}$

From now one we shall denote the curved $C$-space basis generators $\gamma_{M}, \gamma^{M}$ by $E_{M}, E^{M}$, and the flat tangent space generators $\gamma_{A}, \gamma^{A}$ by $E_{A}, E^{A}$. The indices $A, B, C, \ldots$ from the beginning of the alphabet represent the tangent space indices, while those from the middle of the alphabet $L, M, N, \ldots$ represent the base world indices. The covariant derivative of $E_{M}^{A}(\mathbf{X}), E_{A}^{M}(\mathbf{X})$ involves the generalized connection and spin connection and are defined as

$$
\begin{aligned}
\nabla_{K} E_{M}^{A} & =\partial_{K} E_{M}^{A}-\Gamma_{K M}^{L} E_{L}^{A}+\omega_{K B}^{A} E_{M}^{B} \\
\nabla_{K} E_{A}^{M} & =\partial_{K} E_{A}^{M}+\Gamma_{K L}^{M} E_{A}^{L}-\omega_{K A}^{B} E_{B}^{M}
\end{aligned}
$$

If the nonmetricity is zero then $\nabla_{K} E_{M}^{A}=0, \nabla_{K} E_{A}^{M}=0$ in eqs-(2.1).

The coefficients (functions) $W_{L M}^{N}$ associated to the Clifford geometric product are defined by

$$
\begin{gathered}
E_{A} E_{B}=W_{A B}^{C} E_{C}, \text { given } E_{L}=E_{L}^{A} E_{A}, E_{M}=E_{M}^{A} E_{A} \Rightarrow \\
E_{L} E_{M}=W_{L M}^{N} E_{N} \Rightarrow W_{L M}^{N}=E_{L}^{A} E_{M}^{B} E_{C}^{N} W_{A B}^{C}
\end{gathered}
$$

the Clifford algebra structure functions $f_{L M}, d_{L M}^{N}$ are defined by

$$
\begin{gathered}
{\left[E_{A}, E_{B}\right]=f_{A B}^{C} E_{C}, \quad\left[E_{L}, E_{M}\right]=f_{L M}^{N} E_{N} \Rightarrow f_{L M}^{N}=E_{L}^{A} E_{M}^{B} E_{C}^{N} f_{A B}^{C}} \\
\left\{E_{A}, E_{B}\right\}=d_{A B}^{C} E_{C}, \quad\left\{E_{L}, E_{M}\right\}=d_{L M}^{N} E_{N} \Rightarrow d_{L M}^{N}=E_{L}^{A} E_{M}^{B} E_{C}^{N} d_{A B}^{C}
\end{gathered}
$$

Due to the antisymmetry property $\Omega_{K A B}=-\Omega_{K B A}$ of the generalized spin connection one has

$$
\nabla_{K}\left(\eta_{A B}\right)=-\Omega_{K A}^{C} \eta_{C B}-\Omega_{K B}^{C} \eta_{A C}=-\left(\Omega_{K A B}+\Omega_{K B A}\right)=0
$$

as expected and such that

$$
\nabla_{K}\left(g_{M N}\right)=\nabla_{K}\left(E_{M}^{A} E_{N}^{B} \eta_{A B}\right)=0 \Rightarrow \nabla_{K} E_{M}^{A}=0
$$

From

$$
\nabla_{K}\left(E_{M}^{A}\right)=0 \Rightarrow \partial_{K}\left(E_{M}^{A}\right)-\Gamma_{K M}^{L} E_{L}^{A}+\Omega_{K B}^{A} E_{M}^{B}=0 \Rightarrow
$$




$$
\partial_{K}\left(E_{M}^{A}\right)=\Gamma_{K M}^{L} E_{L}^{A}-\Omega_{K B}^{A} E_{M}^{B}
$$

one obtains the relationship between the connection and the spin connection. Having

$$
\begin{gathered}
\nabla_{K}\left(E_{M}^{A}\right)=0 \Rightarrow \nabla_{K}\left(E_{M}\right)=\nabla_{K}\left(E_{M}^{A} E_{A}\right)=E_{M}^{A} \nabla_{K} E_{A}= \\
E_{M}^{A}\left(\partial_{K} E_{A}-\Omega_{K A}^{B} E_{B}\right)=0 \Rightarrow \\
\partial_{K} E_{A}=\Omega_{K A}^{B} E_{B}
\end{gathered}
$$

Hence under parallel transport, $\partial_{K} E_{A}$, the tangent space basis $E_{A}$ generators are rotated as displayed by eq-(2.8). More details of the role of the generalized spin connection in $C$-spaces can be found in [7].

The result $\nabla_{K}\left(E_{M}\right)=0$ is also consistent with the zero nonmetricity condition

$\nabla_{K} g_{M N}=\nabla_{K}<E_{M} E_{N}>=<\nabla_{K}\left(E_{M}\right) E_{N}>+<E_{M} \nabla_{K}\left(E_{N}\right)>=0$

therefore, the Clifford algebra basis elements $E_{M}$ in a curved $C$-space are covariantly constant with respect to a metric-compatible connection $\nabla_{K} g_{M N}=0$.

Upon taking derivatives on both sides of the equalities in eqs-(2.2-2.4) and after using eqs- $(2.7,2.8)$ gives the covariantly constancy conditions of the structure functions

$$
\nabla_{K}\left(f_{L M N}\right)=0, \quad \nabla_{K}\left(d_{L M N}\right)=0, \quad \nabla_{K}\left(W_{L M N}\right)=0
$$

A careful analysis reveals that eqs-(2.13) do not impose any additional constraints on the generalized connection and spin connection. This result is an improvement over our prior findings in [11] and is consistent with the fact that performing a derivative operation on both sides of an equality should not introduce additional constraints on the connection.

For simplicity we shall set the nonmetricity $Q_{M N}^{L}$ to zero. In Appendix B we show that the tor sionless Levi-Civita connection is given by

$$
{ }^{(l c)} \Gamma_{M N}^{L}=\left\{\begin{array}{l}
L \\
M N
\end{array}\right\}+\frac{1}{2} g^{L K}\left(f_{M K N}+f_{N K M}+f_{M N K}\right)
$$

where

$$
\left\{\begin{array}{l}
L \\
M N
\end{array}\right\}=\frac{1}{2} g^{L K}\left(\partial_{N} g_{K M}+\partial_{M} g_{K N}-\partial_{K} g_{M N}\right)
$$

and $f_{M K N}$ are the Clifford algebra structure functions (coefficients). We should notice that the Levi-Civita connection in eq- $(2.14)$ has a symmetric ${ }^{(l c)} \Gamma_{(M N)}^{L}$ and antisymmetric ${ }^{(l c)} \Gamma_{[M N]}^{L}$ piece. The symmetric piece is given by the first three terms in $(2.14)$, while the antisymmetric piece is given by the last term in (2.14).

The Torsion is defined by

$$
\mathbf{T}=\nabla_{\mathbf{X}} \mathbf{Y}-\nabla_{\mathbf{Y}} \mathbf{X}-[\mathbf{X}, \mathbf{Y}]
$$

so that by inspection one can see that the LC connection (2.14) is torsionless

$$
{ }^{(l c)} T_{M N}^{L} \equiv{ }^{(l c)} \Gamma_{M N}^{L}-{ }^{(l c)} \Gamma_{N M}^{L}-f_{M N}^{L}=0
$$


The last term $-f_{M N}^{L}$ in the expression for the torsion (2.17) originates from the nonvanishing $[\mathbf{X}, \mathbf{Y}] \neq 0$ contribution and resulting from the fact that $\left[E_{M}, E_{N}\right]=f_{M N} E_{L} \neq$ 0 .

The Torsion can be introduced explicitly by the addition of the contorsion term $K^{L}{ }_{M N}$

$$
\Gamma_{M N}^{L}={ }^{(l c)} \Gamma_{M N}^{L}+K_{M N}^{L}
$$

The contorsion tensor is defined in terms of the components of the torsion tensor as

$$
K_{M N}^{L}=\frac{1}{2}\left(T_{M}^{L}{ }_{N}+T_{N}^{L}{ }_{M}+T_{M N}^{L}\right), T_{M N}^{L}=-T_{N M}^{L}
$$

so that now the torsion is no longer zero $T_{M N}^{L}=\Gamma_{M N}^{L}-\Gamma_{N M}^{L}-f_{M N}^{L} \neq 0$.

After recurring to the result in eq-(2.7) $\partial_{K}\left(E_{M}^{A}\right)=\Gamma_{K M}^{L} E_{L}^{A}-\Omega_{K B}^{A} E_{M}^{B}$ and defining $T_{M N}^{L} E_{L}^{A}=T_{M N}^{A}$, one can verify that

$$
T_{M N}^{A}=\partial_{M} E_{N}^{A}-\partial_{N} E_{M}^{A}+\Omega_{M B}^{A} E_{N}^{B}-\Omega_{N B}^{A} E_{M}^{B}-f_{M N}^{L} E_{L}^{A}
$$

therefore, $T_{M N}^{A}$ can be written in terms of the generalized spin connection and the generalized vielbeins. The expression (2.20) bears a resemblance with the Cartan structure equations for the torsion 2 -form $\mathbf{T}^{a}=T_{\mu \nu}^{a} d x^{\mu} \wedge d x^{\nu}$ in ordinary spaces when it is written in terms of differential forms, exterior derivatives and exterior products

$$
\mathbf{T}^{a}=\mathbf{d} \Theta^{a}+\omega_{b}^{a} \wedge \Theta^{b}, \Theta^{a} \equiv e_{\nu}^{a} d x^{\nu}, \quad \omega_{b}^{a}=\omega^{a}{ }_{b \mu} d x^{\mu}
$$

The curvature is defined as

$$
\mathbf{R}(\mathbf{X}, \mathbf{Y}) \mathbf{Z}=\left[\nabla_{\mathbf{X}}, \nabla_{\mathbf{Y}}\right] \mathbf{Z}-\nabla_{[\mathbf{X}, \mathbf{Y}]} \mathbf{Z}
$$

the explicit curvature components are then given by

$$
\mathbf{R}_{M N J}^{K}=\partial_{M} \Gamma_{N J}^{K}-\partial_{N} \Gamma_{M J}^{K}-\Gamma_{M J}^{L} \Gamma_{N L}^{K}+\Gamma_{N J}^{L} \Gamma_{M L}^{K}-f_{M N}^{L} \Gamma_{L J}^{K}
$$

To sum up, because the Clifford algebra structure functions (coefficients) are not zero $f_{M N}^{L} \neq 0$ one must include them into the definitions of the torsion and curvature. In the curvature case there are terms $f_{M N}^{L} \Gamma_{L J}^{K}$ as displayed in eq-(2.23). While in the torsion case we must include the term $f_{M N} L$ as shown in $(2.17,2.20)$.

The same-grade $C$-space metric components obeying $g_{M N}=g_{N M}$ are of the form

$$
g_{\mathbf{0 0}}, \quad g_{\mu \nu}, \quad g_{\mu_{1} \mu_{2} \nu_{1} \nu_{2}}, \ldots, g_{\mu_{1} \mu_{2} \ldots \mu_{D} \nu_{1} \nu_{2} \ldots \nu_{D}}
$$

In the most general case the metric does not factorize into antisymmetrized sums of products of the form

$$
\begin{gathered}
g_{\left[\mu_{1} \mu_{2}\right]\left[\nu_{1} \nu_{2}\right]}\left(x^{\mu}\right) \neq g_{\mu_{1} \nu_{1}}\left(x^{\mu}\right) g_{\mu_{2} \nu_{2}}\left(x^{\mu}\right)-g_{\mu_{2} \nu_{1}}\left(x^{\mu}\right) g_{\mu_{1} \nu_{2}}\left(x^{\mu}\right) \\
g_{\left[\mu_{1} \mu_{2} \ldots \mu_{k}\right]\left[\nu_{1} \nu_{2} \ldots \nu_{k}\right]}\left(x^{\mu}\right) \neq \operatorname{det} G_{\mu_{i} \nu_{j}}=\epsilon^{j_{1} j_{2} \ldots j_{k}} g_{\mu_{1} \nu_{j_{1}}} g_{\mu_{2} \nu_{j_{2}}} \ldots g_{\mu_{2} \nu_{j_{k}}}, \quad k=1,2,3, \ldots D
\end{gathered}
$$


The determinant of $G_{\mu_{i} \nu_{j}}$ can be written as

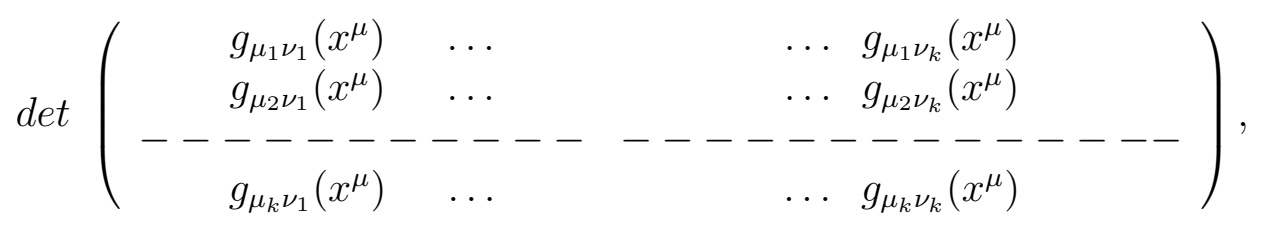

The metric component $g_{\mathbf{0 0}}$ involving the scalar "directions" in $C$-space of the Clifford polyvectors must also be included. It behaves like a Clifford scalar. The other component $g_{\left[\mu_{1} \mu_{2} \ldots \mu_{D}\right]\left[\nu_{1} \nu_{2} \ldots \nu_{D}\right]}$ involves the pseudo-scalar "directions". The latter scalar and pseudoscalars might bear some connection to the dilaton and axion fields in Cosmology and particle physics.

The Bianchi identities are

$$
\begin{gathered}
\mathbf{R}_{M N J}^{K}+\mathbf{R}_{N J M}^{K}+\mathbf{R}_{J M N}^{K}=0 \\
\nabla_{L}\left(\mathbf{R}_{M N J}^{K}\right)+\nabla_{M}\left(\mathbf{R}_{N L J}^{K}\right)+\nabla_{N}\left(\mathbf{R}_{L M J}^{K}\right)=0
\end{gathered}
$$

To finalize this section we shall discuss the notion of poly-differential forms. In $C$-space one has now that

$$
d x^{\mu \nu} \neq d x^{\mu} \wedge d x^{\nu}, \quad d x^{\mu \nu \rho} \neq d x^{\mu} \wedge d x^{\nu} \wedge d x^{\rho}, \ldots
$$

because the areal-coordinates $x^{\mu \nu}$, volume-coordinates $x^{\mu \nu \rho}, \ldots$. associated with the world-sheet, world-volume, ..... evolution of a string, membrane, ..... are not related to the vector coordinates $x^{\mu}$ associated with the evolution of a point particle. For this reason the antisymmetry property of the poly-differential forms is given by $d X^{M} \wedge d X^{N}=-d X^{N} \wedge$ $d X^{M}$. In particular one has the following combinations

$$
\begin{aligned}
d x^{\mu_{1} \mu_{2} \ldots \mu_{2 m}} \wedge d x^{\rho_{1} \rho_{2} \ldots \rho_{2 n}}=-d x^{\rho_{1} \rho_{2} \ldots \rho_{2 n}} \wedge d x^{\mu_{1} \mu_{2} \ldots \mu_{2 m}} \\
d x^{\mu_{1} \mu_{2} \ldots \mu_{2 m-1}} \wedge d x^{\rho_{1} \rho_{2} \ldots \rho_{2 n-1}}=-d x^{\rho_{1} \rho_{2} \ldots \rho_{2 n-1}} \wedge d x^{\mu_{1} \mu_{2} \ldots \mu_{2 m-1}} \\
d x^{\mu_{1} \mu_{2} \ldots \mu_{2 m-1}} \wedge d x^{\rho_{1} \rho_{2} \ldots \rho_{2 n}}=-d x^{\rho_{1} \rho_{2} \ldots \rho_{2 n}} \wedge d x^{\mu_{1} \mu_{2} \ldots \mu_{2 m-1}} \\
d x^{\mu_{1} \mu_{2} \ldots \mu_{2 m}} \wedge d x^{\rho_{1} \rho_{2} \ldots \rho_{2 n-1}}=-d x^{\rho_{1} \rho_{2} \ldots \rho_{2 n-1}} \wedge d x^{\mu_{1} \mu_{2} \ldots \mu_{2 m}}
\end{aligned}
$$

and which differs from the antisymmetry property of ordinary differential forms. Given an ordinary $p$-form $\mathbf{A}_{p}$ and an ordinary $q$-form $\mathbf{B}_{q}$ one has $\mathbf{A}_{p} \wedge \mathbf{B}_{q}=(-1)^{p q} \mathbf{B}_{q} \wedge \mathbf{A}_{p}$. The antisymmetry property displayed by the $C$-space poly-differential forms in eqs- $(2.29,2.30)$ will ensure that the generalized curvature tensor is antisymmetric under the following exchange of polyvector-valued indices : $R_{M N J}^{K}=-R_{N M J}^{K}$.

The $C$-space poly-differential forms analogs of the Cartan-structure equations in ordinary spacetime are 


$$
\begin{array}{r}
\mathbf{T}^{A}=\mathbf{d} \Theta^{A}+\Omega_{B}^{A} \wedge \Theta^{B}, \Theta^{A} \equiv E_{M}^{A} d X^{M}, \quad \Omega_{B}^{A}=\Omega_{B N}^{A} d X^{N} \\
\mathbf{R}^{A B}=\mathbf{d} \Omega^{A B}+\Omega^{A}{ }_{C} \wedge \Omega^{C B}, \quad \mathbf{R}^{A B}=\mathbf{R}_{M N}^{A B} d X^{M} \wedge d X^{N}
\end{array}
$$

where $A, B$ are the tangent space indices and $M, N$ are the base (world) indices.

The above equations are the starting point to formulate a gauge theory of extended gravity in $C$-spaces based on the analogs of a vielbein $E_{M}^{A}$ and spin connection $\Omega_{M}^{A B}$. The generalized connection is $\mathbf{A}_{M}=E_{M}^{A} P_{A}+\Omega_{M}^{A B} J_{A B}$. $P_{A}$ is the translation generator and $J_{A B}$ is the generalized Lorentz generator. The connection poly-differential one-form is $\mathbf{A}_{M} d X^{M}$ and the poly-differential curvature 2 -form is $\mathbf{R}=(\mathbf{d}+\mathbf{A}) \wedge \mathbf{A}$. In component form, the curvature is $\mathbf{R}_{M N} d X^{M} \wedge d X^{N}=\left(\mathbf{R}_{M N}^{A} P_{A}+\mathbf{R}_{M N}^{A B} J_{A B}\right) d X^{M} \wedge d X^{N}$. This gauge theory approach to $C$-space gravity will be the subject of a future investigation and is the $C$-space generalization of the Poincare gauge theory formulation of ordinary gravity [12].

\section{The Generalized Field Equations and the Cosmo- logical Constant}

Before embarking into this final section we shall work with the natural units $\hbar=c=G=$ $L_{\text {Planck }}=1$. Upon performing contractions of the curvature yields the analog of the Ricci tensor $\delta_{K}^{N} \mathbf{R}_{M N J}^{K}=\mathbf{R}_{M J}$ and the Ricci scalar $g^{M J} \mathbf{R}_{M J}=\mathbf{R}$. One may then construct an Einstein-Hilbert-Cartan like action based on the $C$-space curvature scalar $R$

$$
\begin{gathered}
\frac{1}{2 \kappa^{2}} \int d s \prod d x^{\mu} \prod d x^{\mu_{1} \mu_{2}} \ldots d x^{\mu_{1} \mu_{2} \ldots \mu_{D}} \mu_{m}\left(g_{M J}\right) \quad \mathbf{R} \equiv \\
\frac{1}{2 \kappa^{2}} \int[\mathbf{D X}] \mu_{m}\left(g_{M J}\right) \mathbf{R}
\end{gathered}
$$

where $\mu_{m}\left(g_{M J}\right)$ is a suitable integration measure and $\kappa^{2}$ is the gravitational coupling constant in the $2^{D}$-dimensional $C$-space.

At this point it is important to remark that the analog of the Ricci tensor $\mathbf{R}_{M J} \neq \mathbf{R}_{J M}$ is no longer symmetric in the indices because $\mathbf{R}_{M J}$ (and $\mathbf{R}$ ) are defined now in terms of the non-symmetric connection $\Gamma_{M N}^{K} \neq \Gamma_{N M}^{K}$ as displayed in eq-(2.14). There is an antisymmetric piece in the connection given explicitly by the very last term of eq-(2.14). The curvature scalar becomes $\mathbf{R}=g^{M J} \mathbf{R}_{M J}=g^{M J} \mathbf{R}_{(M J)}+g^{M J} \mathbf{R}_{[M J]}=g^{M J} \mathbf{R}_{(M J)}$. Hence, it is the symmetric part of the Ricci tensor analog that appears in the vacuum field equations below. Torsion can also be added to the connection explicitly in terms of the contorsion terms as shown in eqs- $(2.18,2.19)$.

In a given coordinate system (a generalized Lorentz frame) the mixed-grade compo-

nents of the metric $g_{M N}, g^{M N}$, beins $E_{M}^{A}$, inverse beins $E_{A}^{M}$, can be set to zero in order to considerably simplify the calculations; i.e. namely due to the very large diffeomorphism 
symmetry in $C$-space, one may choose a frame ("diagonal gauge") such that the mixed grade components of the metric $g_{M N}$, beins $E_{M}^{A}$, inverse beins $E_{A}^{M}$ are zero. In this case the $C$-space metric components can be chosen to be given by the determinant expressions in eq- $(2.26)$.

The advantage of having $g_{M N}=0$ if the grade of $M$ is not the same as the grade of $N$ is that the determinant of the $C$-space metric can be factorized as the product of determinants of matrices which are comprised of entries given themselves by determinants $(2.26)$ . If an ordering prescription of indices is introduced, $\mu_{1}<\mu_{2}<\ldots \mu_{n}$ and $\nu_{1}<\nu_{2}<\ldots \nu_{n}$, the bivector-bivector components of the $C$-space metric in $D=4$ dimensions $g_{\mu_{1} \mu_{2} \nu_{1} \nu_{2}}$ can be arranged into an ordered square array of entries given by a $6 \times 6$ matrix, since the number of independent bivector components in $D=4$ is $4 \times 3 / 2=6$. For instance, the entries of the square $6 \times 6$ matrix $g_{\mu_{1} \mu_{2} \nu_{1} \nu_{2}}$ are given themselves by determinants : $g_{1212}=g_{11} g_{22}-g_{12} g_{21} ; g_{1313}=g_{11} g_{33}-g_{13} g_{31}, \ldots \ldots$ etc, and such that its determinant is given by the ordinary determinant of an square $6 \times 6$ matrix.

The trivector-trivector components of the $C$-space metric in $D=4$ dimensions $g_{\mu_{1} \mu_{2} \mu_{3} \nu_{1} \nu_{2} \nu_{3}}$ can be arranged into an ordered square array of entries given by a $4 \times 4$ matrix, since the number of independent trivector components in $D=4$ is $4 \times 3 \times 2 / 2 \times 3=4$. The entries of this square $4 \times 4$ matrix are given themselves by the determinants as shown in eq-(2.26). Following a similar procedure with the other $C$-space metric components, in this way one can write the measure of integration in $D=4$ as the square root of the product of determinants

$$
\mu_{m}\left(g_{M J}\right)=\sqrt{|g|\left|\operatorname{det}\left(g_{\mu \nu}\right)\right|\left|\operatorname{det}\left(g_{\mu_{1} \mu_{2} \nu_{1} \nu_{2}}\right)\right|\left|\operatorname{det}\left(g_{\mu_{1} \mu_{2} \mu_{3} \nu_{1} \nu_{2} \nu_{3}}\right)\right|\left|\operatorname{det}\left(g_{\mu_{1} \mu_{2} \mu_{3} \mu_{4} \nu_{1} \nu_{2} \nu_{3} \nu_{4}}\right)\right|}
$$

where $g$ is the scalar-scalar part of the $C$-space metric. The generalization to other dimensions is straightforward.

In the most general case one can have a $C$-space metric with non-vanishing mixed grade components such that the metric $g_{M J}$ components can be assembled into arrays of ordered rectangular matrices. The problem becomes that one cannot longer define a determinant of a rectangular matrix. One can also view the $g_{M J}$ as a hyper-matrix but the construction (if possible) of the hyper-determinant of the $C$-space metric (a hyper matrix) is a more difficult problem [14], [15].

Despite that in the most general case the measure $\mu_{m}\left(g_{M J}\right)$ is not given by eq- $(3.2)$ one can still assume that $\mu_{m}\left(g_{M J}\right)$ is a suitable measure of integration obeying the condition

$$
\delta \mu_{m}\left(g_{M J}\right)=-\frac{1}{2} \mu_{m}\left(g_{M J}\right) g_{M J} \delta g^{M J}
$$

and which is similar to the variational behavior of the square root of an ordinary determinant of the spacetime metric $\sqrt{\left|\operatorname{det} g_{\mu \nu}\right|}$.

Therefore, when the torsion is set to zero and the measure obeys (3.3), a variation of the action (3.1) leads to

$$
\int[\mathbf{D X}] \mu_{m}\left(g_{M J}\right)\left(\mathbf{R}_{(M J)}-\frac{1}{2} g_{M J} \mathbf{R}\right) \delta g^{M J}=0
$$


after discarding the total derivative terms that do not contribute to the variation of the action when the variation of the fields vanishes at the boundaries. These total derivative terms stem from the variation of the Ricci tensor and which after a lengthy but straightforward algebra is given by ${ }^{1}$

$$
\delta\left(\mathbf{R}_{M J}\right)=\nabla_{M}\left(\delta \Gamma_{N J}^{N}\right)-\nabla_{N}\left(\delta \Gamma_{M J}^{N}\right)+2 \Gamma_{[M L]}^{N} \delta\left(\Gamma_{N J}^{L}\right)-\delta\left(f_{M L}^{N} \Gamma_{N J}^{L}\right)
$$

when the torsion is zero one has $2 \Gamma_{[M L]}^{N}=f_{M L}^{N}$ such that eq-(3.5a) becomes

$$
\delta\left(\mathbf{R}_{M J}\right)=\nabla_{M}\left(\delta \Gamma_{N J}^{N}\right)-\nabla_{N}\left(\delta \Gamma_{M J}^{N}\right)-\delta\left(f_{M L}^{N}\right) \Gamma_{N J}^{L}
$$

Because $\Gamma_{N J}^{L}$ does not behave like a tensor the term $\delta\left(f_{M L}^{N}\right) \Gamma_{N J}^{L}$ in eq- $(3.5 \mathrm{~b})$ is spurious unless one is forced to impose the condition $\delta\left(f_{M L}^{N}\right)=0$ and whose physical interpretation is that one does not vary the Clifford algebraic structure functions in $C$-space. Therefore, when one sets $\delta\left(f_{M L}{ }^{N}\right)=0$, the variation $\delta\left(\mathbf{R}_{M J}\right)$ in eq-(3.5b) becomes finally

$$
\delta\left(\mathbf{R}_{M J}\right)=\nabla_{M}\left(\delta \Gamma_{N J}^{N}\right)-\nabla_{N}\left(\delta \Gamma_{M J}^{N}\right)
$$

and which is the analog of the Palatini identity in $C$-space. The variation (3.5c) contributes to a sum of total derivatives by noticing that one can pull the $\nabla$ derivatives to the left of all the terms in the integrand because $\nabla_{K} g_{M J}=0$ and $\nabla \mu_{m}\left(g_{M J}\right)=0$ when the nonmetricity is zero. This yields finally $\int \nabla\left(\mu_{m} \ldots \ldots \delta \Gamma\right)$, which is a total derivative leading then to a boundary term that vanishes, either by imposing a zero variation at the boundaries and/or by having the fields vanish at infinity.

Finally, the vacuum field equations in $C$-space are given by

$$
\mathbf{R}_{(M J)}-\frac{1}{2} g_{M J} \mathbf{R}=0
$$

One must supplement the above equations with the variation of the action with respect to the scalar-scalar component $g$ of the $C$-space metric $\delta S / \delta g=0$.

If there is torsion due to the presence of spinning matter in the Lagrangian one has extra terms

$$
\frac{1}{2 \kappa^{2}} \int[\mathbf{D X}] \mu\left(g_{M J}\right) g^{M J} T_{M N}^{L} \delta \Gamma_{L J}^{N}
$$

in the variation of the action that are matched with the variation $-\delta S_{\text {matter }}$ of the matter terms, if and only if, the variation $\delta \Gamma_{L J}^{N}$ is taken to be independent of the variation $\delta g^{M N}$. One has then

$$
\frac{1}{2 \kappa^{2}} \int[\mathbf{D X}] \mu\left(g_{M J}\right) g^{M J} T_{M N}^{L}=-\frac{\delta S_{m a t t e r}}{\delta \Gamma_{L J}^{N}}
$$

\footnotetext{
${ }^{1}$ After using $\nabla_{M}\left(\delta \Gamma_{N J}^{N}\right)=\partial_{M}\left(\delta \Gamma_{N J}^{N}\right)-\Gamma_{M J}^{L} \delta \Gamma_{N L}^{N} ; \nabla_{N}\left(\delta \Gamma_{M J}^{N}\right)=\partial_{N}\left(\delta \Gamma_{M J}^{N}\right)-\Gamma_{N M}^{L} \delta \Gamma_{L J}^{N}-\Gamma_{N J}^{L} \delta \Gamma_{M L}^{N}+$ $\Gamma_{N L}^{N} \delta \Gamma_{M J}^{L}$
} 
One must also add the contribution of the stress energy tensor $\kappa^{2} \mathbf{T}_{M J}$ to the right hand side of eq-(3.6) and which is given by

$$
\mathbf{T}_{M J} \equiv-\frac{2}{\mu\left(g_{M J}\right)} \frac{\delta\left(\mu\left(g_{M J}\right) \mathcal{L}_{\text {matter }}\right)}{\delta g^{M J}}
$$

We have arrived now at the most salient physical feature of the vacuum field equations. By inserting the torsionless connection expression in eq- $(2.14)$ of the form $\Gamma_{M N}^{L}=\left\{\begin{array}{l}L \\ M N\end{array}\right\}+$ $f_{M N}^{L} \ldots .$. terms, and after using the covariantly constancy condition on the curved $C$-space Clifford algebra structure functions $\nabla_{M} f_{J K L}=0$, one can decompose the Ricci tensor as $\mathbf{R}_{(M J)} \sim R_{M J}+f_{M}^{K L} f_{K L J}+f_{J}^{K L} f_{K L M}$, and the Ricci scalar as $\mathbf{R} \sim R+f^{J K L} f_{J K L}$. $R_{M J}=R_{J M}, R$ are the Ricci tensor and Ricci scalar analogs in $C$-space associated with the symmetric Christoffel connection $\left\{\begin{array}{l}L_{M N} \\ M N\end{array}\right\}=\left\{\begin{array}{l}L_{N M} \\ N\end{array}\right.$.

The physical significance of this curvature decomposition is that these extra terms involving the curved $C$-space Clifford algebra structure functions can be interpreted as an effective stress energy tensor which can mimic the effects of "dark" matter/energy. To see how the cosmological constant $\Lambda$ emerges, it is straightforward to infer that the contraction $f^{J K L} f_{J K L}$ involving the Clifford-algebra structure functions in curved $C$ space turns out to be equal to $f^{A B C} f_{A B C} \sim \Lambda_{1}=$ constant, when $f^{A B C}, f_{A B C}$ are the tangent space Clifford algebra structure constants. This finding is just a consequence of the definitions of $f^{J K L}$ and $f_{J K L}$ in terms of the beins $E_{J}^{A}$, and inverse beins $E_{A}^{J}$ given by eqs-(2.2-2.4), and obeying $E_{A}^{J} E_{M}^{A}=\delta_{M}^{J}, \ldots$

Therefore, when the torsion is set to zero, the measure obeys (3.3), and after writing $\mathbf{R}_{(M J)}=R_{(M J)}+\Delta R_{(M J)}$, and $\mathbf{R}=R+\Lambda_{1}$, the vacuum field equations in $C$-space can be rewritten as

$$
R_{(M J)}+\Delta R_{(M J)}-\frac{1}{2} g_{M J} R-\frac{1}{2} g_{M J} \Lambda_{1}=0
$$

where $\Lambda_{1} \equiv \Delta R \sim f^{J K L} f_{J K L}=f^{A B C} f_{A B C}=$ constant. The other terms

$$
\Delta R_{(M J)} \sim f_{M}^{K L} f_{K L J}+f_{J}^{K L} f_{K L M} \sim \Lambda_{2} \kappa_{M J}
$$

are proportional to the curved space Clifford algebra Killing metric $\kappa_{M J}=$ $E_{M}^{A} E_{J}^{D} f_{A}^{B C} f_{B C D}=E_{M}^{A} E_{J}^{D} \kappa_{A D}$. If the Killing metric $\kappa_{A D}$ coincides with $\eta_{A D}$ then $\kappa_{M J}=g_{M J}$ and the combined effect of the two constants $\Lambda_{1}, \Lambda_{2}$ gives the sought-after cosmological constant term

$$
\frac{1}{2} g_{M J}\left(2 \Lambda_{2}-\Lambda_{1}\right) \equiv \Lambda g_{M J}, \text { with } 2 \Lambda_{2}-\Lambda_{1} \equiv 2 \Lambda
$$

If the Killing metric $\kappa_{A D}$ does not coincide with $\eta_{A D}$ then one will have for the $\Delta R_{(M J)}$ terms the following $\Lambda_{2} \kappa_{M J}$ contribution which can be interpreted as (minus) an effective stress energy tensor $-\kappa^{2} T_{M J}$ term mimicking the effects of "dark" matter.

To conclude, one of the most salient physical feature of the extended gravitational theory in $C$-spaces is that one can generate an effective stress energy tensor mimicking the effects of "dark" matter/energy. In particular the cosmological constant term. One could explicitly add a cosmological constant term, by hand, to the original action (3.1) 
but the main point of our above argument is that it is not necessary, to do so. The cosmological constant term is automatically encoded in the $f^{J K L} f_{J K L}=f^{A B C} f_{A B C}(=$ constant) term which naturally forms part of the $C$-space scalar curvature.

In ordinary Relativity, when the torsion is zero, one can construct the Einstein tensor by performing two successive contractions of the differential Bianchi identity [13]. It also leads to the conservation of the stress energy tensor in the right hand side. In $C$-space the differential Bianchi identities (2.27) are satisfied when the torsion is zero. By performing two successive contractions of the differential Bianchi identities one also arrives at the field equations

$$
\nabla^{M}\left(\mathbf{R}_{(M J)}-\frac{1}{2} g_{M J} \mathbf{R}\right)=0 \Rightarrow \mathbf{R}_{(M J)}-\frac{1}{2} g_{M J} \mathbf{R}=\kappa^{2} \mathbf{T}_{M J}, \quad \nabla^{M}\left(\mathbf{T}_{M J}\right)=0
$$

The advantage of recurring to the differential Bianchi identities in $C$-space to derive the field equations (3.11) is that it is not necessary to invoke an action and confront the subtleties in constructing a suitable measure of integration.

One may introduce a cosmological constant as an integration constant $\Lambda^{\prime}$ in the right hand side of eq-(3.11) giving the modified field equations

$$
\mathbf{R}_{(M J)}-\frac{1}{2} g_{M J} \mathbf{R}=\Lambda^{\prime} g_{M J}
$$

After decomposing the curvature terms of the left hand side of eq-(3.12) in the same form as in eq-(3.8), and bringing the term $\Lambda^{\prime} g_{M J}$ into the left hand side, one ends up with an effective cosmological constant term of the form $\left(\Lambda-\Lambda^{\prime}\right) g_{M J}$. Hence a cancellation of the effective cosmological constant is possible when $\Lambda-\Lambda^{\prime}=0$. This scenario for a plausible explanation of the extremely small value of the observed cosmological constant warrants further investigation.

Let us proceed with the vacuum field equations (3.6). To simplify matters we shall only consider the action (3.1) whose measure is given by (3.2) involving the $C$-space metric components and whose entries are given by the determinant expressions (2.26). Namely, the $C$-space metric is being decomposed into antisymmetrized sums of products of the ordinary metric components of spacetime. Besides the scalar-scalar component $g$ of the $C$-space metric (not to be confused with the $\mid$ det $g_{\mu \nu} \mid$ ), the other independent variables are now given by the ordinary metric components $g_{\mu \nu}=g_{\nu \mu}$, hence a variation of the action in $C$-space with respect to $g_{\mu \nu}$ leads to the generalized vacuum field equations that do not coincide with the Einstein vacuum field equations.

Hence, in the torsionless case, and in this simplified case, the vacuum field equations in $D=4$ are obtained from the variation of the action with respect to $g^{\mu \nu}$ after using the chain rule of differentiation

$$
\begin{gathered}
\int[\mathbf{D X}] \mu_{m}\left(g_{M J}\right)\left(\mathbf{R}_{(M J)}-\frac{1}{2} g_{M J} \mathbf{R}\right) \delta g^{M J}= \\
\int[\mathbf{D X}] \mu_{m}\left(g_{M J}\right)\left(\mathbf{R}_{(M J)}-\frac{1}{2} g_{M J} \mathbf{R}\right) \frac{\delta g^{M J}}{\delta g^{\mu \nu}} \delta g^{\mu \nu}=0
\end{gathered}
$$


The above variation in (3.13) yields the simplified version of the vacuum field equations

$$
\mathbf{R}_{(\mu \nu)}-\frac{1}{2} g_{\mu \nu} \mathbf{R}+\left(\mathbf{R}_{(\hat{M} \hat{J})}-\frac{1}{2} g_{\hat{M} \hat{J}} \mathbf{R}\right) \frac{\delta g^{\hat{M} \hat{J}}}{\delta g^{\mu \nu}}=0
$$

where the contributions of the polyvector-components of the $C$-space metric are denoted explicitly by the hatted indices. Clearly, the vacuum field equations (3.14) differ from the Einstein field equations in ordinary spacetime due to the extra terms stemming from Clifford algebraic structure and polyvector-valued contributions to the $C$-space metric. These extra terms, once again, can be interpreted as the contribution of (minus) an effective stress energy tensor $-\kappa^{2} \mathbf{T}_{\mu \nu}^{e f f}$ which could mimic the effects of "dark" matter/energy. As a reminder, one must also include the equation associated with the scalar-scalar component $g$ of the $C$-space metric $\delta S / \delta g=0$. Such scalar-scalar $C$-space metric component might also have cosmological implications like the axion and dilaton.

There are still many challenges ahead to test the viability of the Extended Gravitational Theory in $C$-spaces. Other physical applications of $C$-space gravity were studied in [11] in relationship to higher curvature theories of gravity, like Lanczos-Lovelock-Cartan gravity (with torsion) [10] and to $f(R)$ extended theories of gravity [16]. Our finding that the presence of the cosmological constant, along with a plausible mechanism to explain its extremely small value and/or its cancellation, can be understood from a purely Clifford algebraic and geometric perspective, alone, is very appealing and deserves further investigation. The reader might have noticed a similarity of our expressions for the torsion and curvature to those which appear in a nonholonomic coordinate description of geometry, a la Finsler, for example. Recent cosmological applications of this nonholonomic approach to geometry, and related to the universe accelerated expansion, can be found in [17].

\section{Acknowledgements}

We are indebted to M. Bowers for assistance and to the guest editors (Eduardo BayroCorrochano, Eckhard Hitzer) of this special issue for their very kind invitation and for raising this problem.

\section{APPENDIX A}

In this Appendix we shall write the (anti) commutator relations [8] for the Clifford algebra generators.

$$
\begin{gathered}
\frac{1}{2}\left\{\gamma_{a}, \gamma_{b}\right\}=g_{a b} \mathbf{1} ; \frac{1}{2}\left[\gamma_{a}, \gamma_{b}\right]=\gamma_{a b}=-\gamma_{b a}, a, b=1,2,3, \cdots, m \\
{\left[\gamma_{a}, \gamma_{b c}\right]=2 g_{a b} \gamma_{c}-2 g_{a c} \gamma_{b}, \quad\left\{\gamma_{a}, \gamma_{b c}\right\}=2 \gamma_{a b c}} \\
{\left[\gamma_{a b}, \gamma_{c d}\right]=-2 g_{a c} \gamma_{b d}+2 g_{a d} \gamma_{b c}-2 g_{b d} \gamma_{a c}+2 g_{b c} \gamma_{a d}}
\end{gathered}
$$

In general one has $[8]$ 


$$
\begin{aligned}
& p q=\mathbf{o d d},\left[\gamma_{m_{1} m_{2} \ldots m_{p}}, \gamma^{n_{1} n_{2} \ldots n_{q}}\right]=2 \gamma_{m_{1} m_{2} \ldots m_{p}}^{n_{1} n_{2} \ldots n_{q}}-\frac{2 p ! q !}{2 !(p-2) !(q-2) !} \delta_{\left[m_{1} m_{2}\right.}^{\left[n_{1} n_{2}\right.} \gamma_{\left.m_{3} \ldots \ldots m_{p}\right]}^{\left.n_{3} \ldots n_{q}\right]}+ \\
& \frac{2 p ! q !}{4 !(p-4) !(q-4) !} \delta_{\left[m_{1} \ldots m_{4}\right.}^{\left[n_{1} \ldots n_{4}\right.} \gamma_{\left.m_{5} \ldots \ldots m_{p}\right]}^{\left.n_{5} \ldots . n_{q}\right]}-\ldots \ldots \ldots \ldots \\
& p q=\text { even, }\left\{\gamma_{m_{1} m_{2} \ldots m_{p}}, \gamma^{n_{1} n_{2} \ldots . . n_{q}}\right\}=2 \gamma_{m_{1} m_{2} \ldots m_{p}}^{n_{1} n_{2} \ldots n_{q}}-\frac{2 p ! q !}{2 !(p-2) !(q-2) !} \delta_{\left[m_{1} m_{2}\right.}^{\left[n_{1} n_{2}\right.} \gamma_{\left.m_{3} \ldots \ldots m_{p}\right]}^{\left.n_{3} \ldots n_{q}\right]}+ \\
& \frac{2 p ! q !}{4 !(p-4) !(q-4) !} \delta_{\left[m_{1} \ldots m_{4}\right.}^{\left[n_{1} \ldots n_{4}\right.} \gamma_{\left.m_{5} \ldots \ldots m_{p}\right]}^{\left.n_{5} \ldots n_{q}\right]}-\ldots \ldots \ldots \\
& p q=\text { even, } \quad\left[\gamma_{m_{1} m_{2} \ldots m_{p}}, \gamma^{n_{1} n_{2} \ldots n_{q}}\right]=\frac{(-1)^{p-1} 2 p ! q !}{1 !(p-1) !(q-1) !} \delta_{\left[m_{1}\right.}^{\left[n_{1}\right.} \gamma_{\left.m_{2} \ldots m_{p}\right]}^{\left.n_{2} \ldots n_{q}\right]}- \\
& \frac{(-1)^{p-1} 2 p ! q !}{3 !(p-3) !(q-3) !} \delta_{\left[m_{1} m_{2} m_{3}\right.}^{\left[n_{1} n_{2} n_{3}\right.} \gamma_{\left.m_{4} \ldots \ldots m_{p}\right]}^{\left.n_{4} \ldots . n_{q}\right]}+\ldots \ldots \\
& p q=\text { odd }, \quad\left\{\gamma_{m_{1} m_{2} \ldots . m_{p}}, \gamma^{n_{1} n_{2} \ldots n_{q}}\right\}=\frac{(-1)^{p-1} 2 p ! q !}{1 !(p-1) !(q-1) !} \delta_{\left[m_{1}\right.}^{\left[n_{1}\right.} \gamma_{\left.m_{2} \ldots m_{p}\right]}^{\left.n_{2} \ldots n_{q}\right]}- \\
& \frac{(-1)^{p-1} 2 p ! q !}{3 !(p-3) !(q-3) !} \delta_{\left[m_{1} m_{2} m_{3}\right.}^{\left[n_{1} n_{2} n_{3}\right.} \gamma_{\left.m_{4} \ldots \ldots m_{p}\right]}^{\left.n_{4} \ldots n_{q}\right]}+\ldots \ldots
\end{aligned}
$$

The generalized Kronecker delta is defined as the determinant

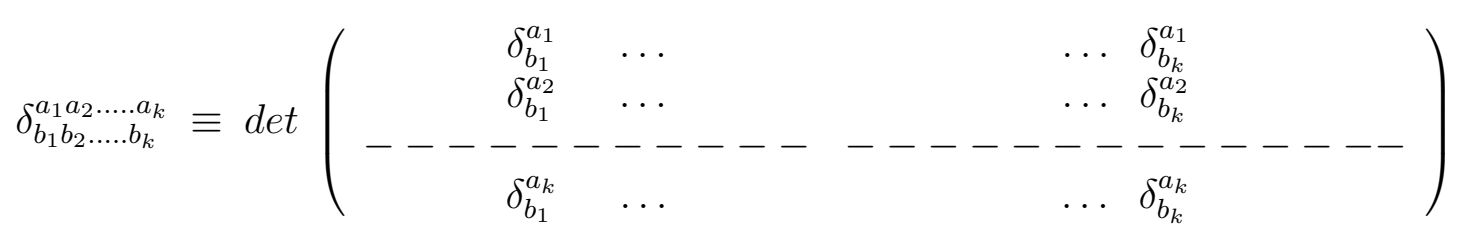

\section{APPENDIX B}

In this appendix we shall derive the expression for the analog of the torsionless LeviCivita connection in $C$-space. Given a symmetric metric $g_{M N}=g_{N M}$ and setting the nonmetricity $Q_{K M N}$ to zero gives

$$
\nabla_{K} g_{M N}=\partial_{K} g_{M N}-\Gamma_{K M}^{L} g_{L N}-\Gamma_{K N}^{L} g_{M L}=0
$$

Performing a cyclic index permutation yields

$$
\nabla_{M} g_{N K}=\partial_{M} g_{N K}-\Gamma_{M N}^{L} g_{L K}-\Gamma_{M K}^{L} g_{N L}=0
$$




$$
\nabla_{N} g_{K M}=\partial_{N} g_{K M}-\Gamma_{N K}^{L} g_{L M}-\Gamma_{N M}^{L} g_{K L}=0
$$

adding eqs-(B.2, B.3) and subtracting eq-(B1) leads to

$$
\partial_{M} g_{N K}+\partial_{N} g_{K M}-\partial_{K} g_{M N}=2 \Gamma_{(M N)}^{L} g_{L K}+2 \Gamma_{[M K]}^{L} g_{L K}+2 \Gamma_{[N K]}^{L} g_{L K}
$$

where

$$
\begin{gathered}
\Gamma_{(M N)}^{L} \equiv \frac{1}{2}\left(\Gamma_{M N}^{L}+\Gamma_{N M}^{L}\right) \\
\Gamma_{[M K]}^{L} \equiv \frac{1}{2}\left(\Gamma_{M K}^{L}-\Gamma_{K M}^{L}\right), \Gamma_{[N K]}^{L} \equiv \frac{1}{2}\left(\Gamma_{N K}^{L}-\Gamma_{K N}^{L}\right)
\end{gathered}
$$

when the Torsion is zero one has

$$
T_{M K}^{L}=\Gamma_{M K}^{L}-\Gamma_{K M}^{L}-f_{M K}^{L}=0 \Rightarrow 2 \Gamma_{[M K]}^{L}=f_{M K}^{L}
$$

such that eq-(B.4) becomes

$$
\partial_{M} g_{N K}+\partial_{N} g_{K M}-\partial_{K} g_{M N}=2 \Gamma_{(M N)}^{L} g_{L K}+f_{M K}^{L} g_{L N}+f_{N K}^{L} g_{L M}
$$

and from eq-(B.8) one can then deduce that the symmetric part of the connection is given by

$$
\Gamma_{(M N)}^{L}=\frac{1}{2} g^{L K}\left[\left(\partial_{M} g_{N K}+\partial_{N} g_{M K}-\partial_{K} g_{M N}\right)+\left(f_{M K N}+f_{N K M}\right)\right]
$$

therefore, by adding the antisymmetric part of the connection $\Gamma_{[M N]}^{L}=\frac{1}{2} f_{M N}^{L}$ to the symmetric part $\Gamma_{(M N)}^{L}$ one obtains finally the full expression for the analog of the torsionless Levi-Civita connection in $C$-space

$$
{ }^{(l c)} \Gamma_{M N}^{L}=\Gamma_{(M N)}^{L}+\Gamma_{[M N]}^{L}=\left\{\begin{array}{l}
L \\
M N
\end{array}\right\}+\frac{1}{2} g^{L K}\left(f_{M K N}+f_{N K M}+f_{M N K}\right)
$$

where

$$
\left\{\begin{array}{l}
L \\
M N
\end{array}\right\} \equiv \frac{1}{2} g^{L K}\left(\partial_{M} g_{N K}+\partial_{N} g_{M K}-\partial_{K} g_{M N}\right)
$$

\section{References}

[1] C. Castro and M. Pavsic, Progress in Physics vol 1 (April 2005) 31. Phys. Letts B 559 (2003) 74. Int. J. Theor. Phys 42 (2003) 1693.

[2] C. Castro, Foundations of Physics 35, no.6 (2005) 971. Prog. in Phys. 1 (April 2005) 20 . 
[3] D. Hestenes, "Spacetime Algebra" Gordon and Breach, New York, 1996.

D. Hestenes and G. Sobcyk, Clifford Algebra to Geometric Calculus (D. Reidel Publishing Company, Dordrecht, 1984).

[4] M. Pavsic, The Landscape of Theoretical Physics: A Global View, from point particles to the brane world and beyond, in search of a Unifying Principle, (Fundamental Theories of Physics, vol. 19, Kluwer Academic Publishers, Dordrecht, Boston, London, 2001).

[5] I. R. Porteous, Clifford Algebras and the Classical Groups (Cambridge University Press, 1995).

[6] W. Baylis, Electrodynamics, A Modern Geometric Approach, Boston (Birkhauser, 1999).

[7] M. Pavsic, "Kaluza-Klein theory without extra dimensions: Curved Clifford space," Phys. Lett. B 614 (2005) 85

"Spin gauge theory of gravity in Clifford space: A Realization of Kaluza-Klein theory in 4-dimensional spacetime," Int. J. Mod. Phys. A 21 (2006) 5905

"On a unified theory of generalized branes coupled to gauge fields, including the gravitational and Kalb-Ramond fields," Found. Phys. 37 (2007) 1197

[8] K. Becker, M. Becker and J. Schwarz, String Theory and M-Theory: A Modern Introduction (Cambridge University Press, 2007, pp. 543-545).

[9] Carlos Castro, "Extended Lorentz Transformations in Clifford Space Relativity Theory "submitted to Advances in Applied Clifford Algebras, September 2014.

[10] C. Lanczos, Annals Math. 39, 842 (1938). D. Lovelock, J. Math. Phys 12 (1971) 498.

[11] Carlos Castro, "Lanczos-Lovelock-Cartan Gravity from Clifford Space Geometry ", Int. J. Geom. Meth. Mod. Phys. 10 (2013) 1350019

"The Extended Relativity Theory in Clifford Phase Spaces and Modifications of Gravity at the Planck/Hubble scales", Adv. Appl. Clifford Algebras 24 (2014) 2953

[12] F. Hehl, P. Von Der Heyde, G. Kerlick and J. Nester, Rev. Mod. Phys 48 (1976) 393.

[13] M. Nakahara, Geometry, Topology and Physics (Institute of Physics Publishing, 1998).

[14] A. Cayley, Cambridge Math. J. 4 (1845) 193.

[15] I. Gelfand, M. Kapranov and A. Zelevinsky, Discriminants, Resultants and Determinants (Birkhauser 1994). 
[16] S. Capozziello and M. De Laurentis, "Extended Theories of Gravity" [arXiv:1108.6266] (to appear in Physics Reports).

[17] S. Vacaru, "Ghost-Free Massive $\mathrm{f}(\mathrm{R})$ Theories Modelled as Effective Einstein Spaces and Cosmic Acceleration", accepted in the European Physical Journal C [Eur. Phys. J.] C 74 (2014); 14 Oct 2014, arXiv: 1401.2882 [physics.gen-ph]. 